\title{
Long-Term Outcome of Adalimumab in a Young Girl with Hidradenitis Suppurativa
}

\author{
Teresa Oranges ${ }^{a, b}$ Andrea Chiricozzi ${ }^{a}$ Michela lannone ${ }^{a}$ Marco Romanelli ${ }^{a}$ \\ Valentina Dini ${ }^{\mathrm{a}}$ \\ a Department of Dermatology, University of Pisa, Pisa, Italy; ${ }^{b}$ Department of Health Sciences, Anna Meyer Children's \\ University Hospital, University of Florence, Florence, Italy
}

\section{Established Facts}

- Adalimumab is approved in adults and children with moderate-to-severe hidradenitis suppurativa (HS).

- Data regarding long-term efficacy and safety in young patients with HS treated with adalimumab are lacking.

\section{Novel Insights}

- We report the long-term outcome in a young patient with HS treated with adalimumab.

\section{Keywords}

Hidradenitis suppurativa · Acne inversa $\cdot$ Children ·

Adalimumab

\section{Abstract \\ Hidradenitis suppurativa (HS) is a chronic, burdensome in- flammatory skin disorder mainly affecting adults with an infrequent onset during childhood or adolescence. Guide- lines regarding the therapeutic drugs in pediatric HS pa- tients are lacking. We describe the case of a 16-year-old girl}

affected by HS with a massive impact of the disease on her daily activities. She underwent antibiotics, estroprogestinics, isotretinoin, and drainage procedures with partial and short-term improvement. We treated the patient with adalimumab and assessed disease severity using BMI, pain VAS, Hurley, m-Sartorius, and HS-PGA. The treatment has been continued for 4 years with a strong improvement of clinical and psychological outcome. No side effects were recorded throughout the treatment period.

(c) 2018 S. Karger AG, Basel

Teresa Oranges, MD

Department of Dermatology, University of Pisa

Via Roma 67

IT-56126 Pisa (Italy)

E-Mail teresa.oranges@gmail.com 


\section{Introduction}

Hidradenitis suppurativa (HS) constitutes a chronic, burdensome inflammatory skin disorder mainly affecting the adult population with an infrequent onset during childhood or adolescence. Early onset is associated with more widespread disease and is more frequent in children with a positive family history $[1,2]$.

Several scores have been used to evaluate the clinical severity of HS or the response to treatments (Hurley, Sartorius, m-Sartorius, PGA, AISI, HSSI, and HiSCR) [3, 4].

Children with HS present hormonal imbalances more frequently than adults do [2].
Topical and systemic antibiotics, antiandrogens, and surgical procedures have been used in children with HS. However, guidelines regarding these therapeutic approaches or including the use of biologic drugs in pediatric HS patients are not currently available $[2,5]$.

\section{Case Report}

We describe the case of a 16-year-old girl presenting with inflamed and noninflamed nodules, with scarring affecting the armpits (Fig. 1a), sternum (Fig. 1b), inguinal regions (Fig. 1c), and submammary folds. The first lesions appeared on the sternum when she was 10 years old, with several recurrences over time.
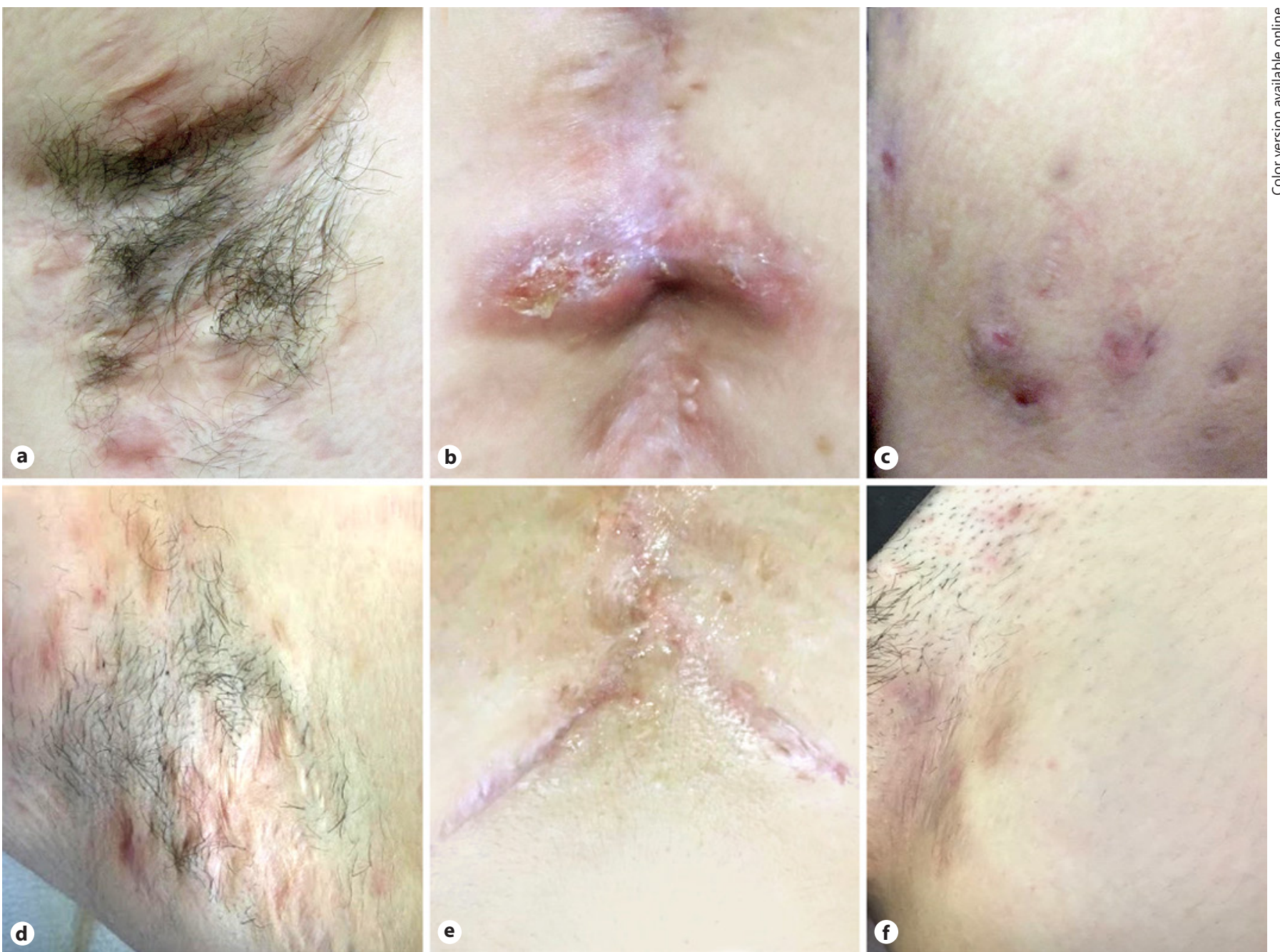

Fig. 1. Inflammatory nodules and scarring affecting the armpit (a), sternum (b), and inguinal region (c) at T0. Residual scarring affecting the armpit (d), sternum (e), and inguinal region (f) at T48, after 4 years of adalimumab, laser treatment on the sternum, and mastopexy. 
Fig. 2. m-Sartorius (a) and HS-PGA (b) score values over time.
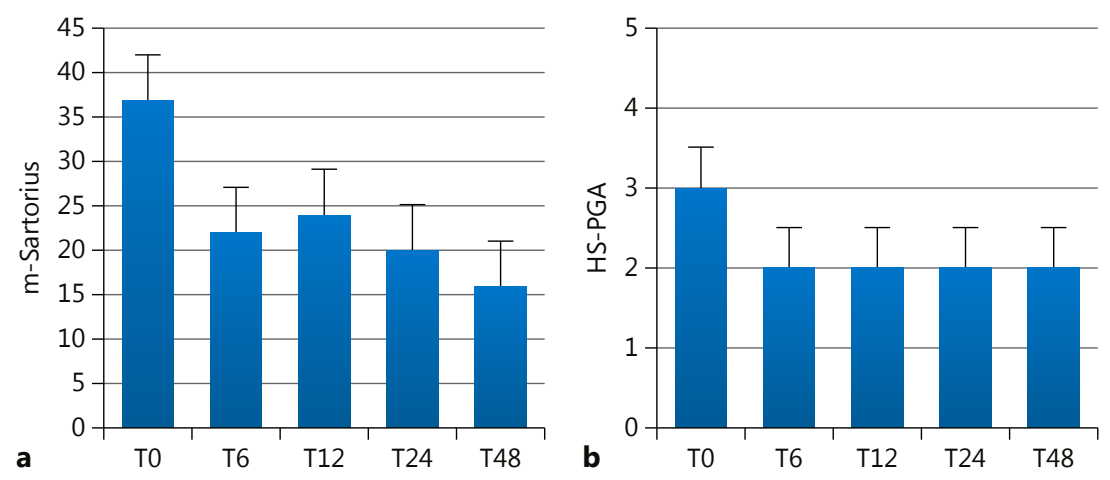

Considering the clinical features of the lesions and the relapsing evolution, we diagnosed HS. She reported a history of acne vulgaris, depression, bulimia, and pilonidal cyst. She had never smoked, and there was no family history of HS. She underwent antibiotics, estroprogestinics, isotretinoin, and drainage procedures with partial and short-term improvement. The impact of the disease on her daily activities was massive, and she missed several days of school due to pain and embarrassment.

We decided to administer adalimumab, although at the time of the event (early 2013) adalimumab had not yet been approved for the treatment of HS, with the agreement of a local pharmacy. Adalimumab $160 \mathrm{mg}$ s.c. was administered at week $0,80 \mathrm{mg}$ at week 2 , and then $40 \mathrm{mg}$ q.w., according to the beneficial response obtained by Kimball et al. [6] in a parallel randomized trial on adalimumab in HS.

We assessed disease severity using BMI, pain VAS, Hurley, mSartorius (Fig. 2a), and HS-PGA (Fig. 2b) at weeks 0 (T0), 6 (T6), 12 (T12), 24 (T24), and 48 (T48).

The treatment has been continued for 4 years with good clinical outcomes (Fig. 1d-f).

After 20 months of treatment, she underwent ND-YAG laser and $\mathrm{CO}_{2}$ laser on the most affected area (the sternum), without discontinuation of adalimumab, obtaining a significant reduction of disease scarring. During the fourth year of treatment, HS-related fibrotic scarring, including retraction and bridge lesions localized on the submammary folds bilaterally, had a profound impact on her quality of life and interpersonal/sexual life; therefore, mastopexy surgery removing fibrotic tissue was performed at that time.

BMI was 37 at $\mathrm{T} 0$ and reduced to 25.1 at T48. Hurley score was III at T0 and reduced to II at T6. Pain VAS was 10 at T0 and showed a rapid, significant reduction, moving to 4 at T6 and varying from 2 to 1 at T12. HS-PGA was moderate at the beginning and became mild at T6, and m-Sartorius reduced from 23 (T0) to 17 (T48) over time.

We observed a strong improvement regarding the psychological impact of the disease and the quality of life of the patient. No side effects were recorded throughout the treatment period. The patient is currently being treated with adalimumab.

\section{Discussion}

HS is characterized by its significant impact on quality of life and its negative effects on daily activities. Early diagnosis and correct therapeutic management, including medical and surgical approaches and postsurgical wound management, are mandatory to reduce relapses and prevent severe complications [3,7].

The therapeutic approach of HS in childhood and adolescents is challenging as, similarly to other skin disorders, recommendations and guidelines exclusively refer to adult patient populations. Thus, guidance of management for child populations is lacking.

In this case, we obtained an almost complete remission of active lesions by using adalimumab.

The goal of the treatment with adalimumab in this disease is to achieve sign and symptom control. However, the complete remission is rarely obtained in patients affected by HS. We achieved a strong reduction of scar tissue by using lasers on limited affected areas and surgery in regions widely involved. This multifaceted therapeutic approach may help in improving quality of life and functionality, and in reducing friction. This is significant because friction may cause worsening of the disease in moderate-to-severe HS unresponsive to traditional systemic treatments.

We reported this case of successful management of HS by combining adalimumab therapy with laser and surgical procedures to achieve an overall control of the disease describing long-term efficacy and safety of adalimumab in an adolescent HS patient. To our knowledge, this treatment period of 4 years is the longest to be reported to date.
Oranges/Chiricozzi/Iannone/Romanelli/ Dini 


\section{Statement of Ethics}

The authors state that the patient has given her informed consent for the pictures, and adalimumab has been used with the agreement of a local pharmacy.

\section{Disclosure Statement}

The authors declare that there are no conflicts of interest and no funding sources for this paper.

\section{References}

1 Palmer RA, Keefe M: Early-onset hidradenitis suppurativa. Clin Exp Dermatol 2001;26: 501-503.

-2 Liy-Wong C, Pope E, Lara-Corrales I: Hidradenitis suppurativa in the pediatric population. J Am Acad Dermatol 2015;73(5 Suppl 1):S36-S41.
3 Zouboulis CC, Desai N, Emtestam L, et al: European $\mathrm{S} 1$ guideline for the treatment of hidradenitis suppurativa/acne inversa. J Eur Acad Dermatol Venereol 2015;29:619-644.

4 Chiricozzi A, Faleri S, Franceschini C, et al: AISI: A new disease severity assessment tool for hidradenitis suppurativa. Wounds 2015; 27:258-264.

5 Mikkelsen PR, Jemec GB: Hidradenitis suppurativa in children and adolescents: a review of treatment options. Paediatr Drugs 2014;16: 483-489.
Kimball AB, Kerdel F, Adams D, et al: Adalimumab for the treatment of moderate to severe hidradenitis suppurativa: a parallel randomized trial. Ann Intern Med 2012;157: 846-855.

7 Dini V, Oranges T, Rotella L, Romanelli M: Hidradenitis suppurativa and wound management. Int J Low Extrem Wounds 2015;14: 236-244. 